\title{
Research on Multistage Rotor Assembly Optimization Methods for Aeroengine Based on the Genetic Algorithm
}

\author{
Yue Chen $\mathbb{D}^{1,2}$ Jiwen Cui $\mathbb{D}^{1,2}$ Xun Sun $\mathbb{D}^{1,2}$ and Shihai Cui ${ }^{3}{ }^{3}$ \\ ${ }^{1}$ Centre of Ultra-precision Optoelectronic Instrument Engineering, Harbin Institute of Technology, Harbin 150080, China \\ ${ }^{2}$ Key Lab of Ultra-precision Intelligent Instrumentation (Harbin Institute of Technology), \\ Ministry of Industry and Information Technology, Harbin 150080, China \\ ${ }^{3}$ College of Mechanical Engineering, Tianjin University of Science \& Technology, Tianjin 300222, China \\ Correspondence should be addressed to Jiwen Cui; cuijiwen@hit.edu.cn
}

Received 6 August 2020; Revised 16 November 2020; Accepted 2 March 2021; Published 23 March 2021

Academic Editor: Kailong Liu

Copyright (c) 2021 Yue Chen et al. This is an open access article distributed under the Creative Commons Attribution License, which permits unrestricted use, distribution, and reproduction in any medium, provided the original work is properly cited.

\begin{abstract}
The coaxiality and unbalance are the two important indexes to evaluate the assembly quality of an aeroengine. It often needs to be tested and disassembled repeatedly to meet the double-objective requirements at the same time. Therefore, an intelligent assembly method is urgently needed to directly predict the optimal assembly orientations of the rotors at each stage to meet the doubleobjective requirements simultaneously. In this study, an assembly optimization method for the multistage rotor of an aeroengine is proposed based on the genetic algorithm. Firstly, a spatial location propagation model is developed to accurately predict the spatial position of each rotor after assembly. The alignment process of the assembly screw holes of the adjacent rotors is considered for the first time. Secondly, a new assembly optimization strategy is proposed to select different assembly data for the specific values of the coaxiality and unbalance, respectively. Finally, a double-objective fitness function is constructed based on the coaxiality and unbalance. The simulation and experimental results show that the assembly optimization method proposed in this study can be utilized to achieve synchronous optimization of the coaxiality and unbalance of an aeroengine during preassembly.
\end{abstract}

\section{Introduction}

The assembly technique is the ultimate technical link during the manufacturing process of an aeroengine. Especially for the core parts typically represented by the high pressure compressor (HPC) and the low pressure compressor (LPC), their assembly quality may have a direct effect on the stability of an aeroengine in high-speed operation [1-3]. Coaxiality and unbalance out of tolerance caused by improper assembly may give rise to complicated vibrations and noises [4-7]. In the actual assembly process of an aeroengine, testing and dismounting need to be repeatedly conducted in most cases, making sure that the two indexes of the coaxiality and unbalance can be satisfied simultaneously $[8,9]$. Without a doubt, such a cumbersome process requires a long time and also a high cost. Therefore, reasonable assembly error control approaches and assembly optimization methods should be developed to meet the double-objective requirements during preassembly. This is particularly critical for the improvement of assembly efficiency and the qualification rate of one-time assembly.

In recent years, both assembly error propagation model and assembly strategy had been extensively investigated by scholars on rotating machinery (e.g., the aeroengine). According to the principles of coordinate transformation, an assembly error propagation model was derived for $2-\mathrm{D}$ rotating rigid bodies by Hussain et al. [10]. For the first time, they came up with a strategy of "straight-build assembly." According to Yang et al. [11], for the purpose of optimizing the standard deviation of eccentric errors for a multistage rotor, eccentric errors were calculated based on a normal axis across the center of a rotor at the bottom and a line from the center of the rotor at the bottom to that of another rotor on the top. By constructing a probability density function for the cumulative eccentric errors of the last-stage rotor, Yang et al. [12] compared the global optimization results obtained 
by probability and Monte Carlo methods, respectively. Their results were proved to be highly consistent with each other, further reflecting the validity of "straight-build assembly." Targeted at a 2-D rotating rigid body model, Yang et al. [13] explored the optimization effects of five different assembly strategies. Based on the above 2-D assembly error propagation model, Hussain et al. [14] proposed another strategy of "parallelism-build assembly." Moreover, such a 2-D assembly error propagation model was further modified into a 3-D model by Yang et al. [12]. Then, in combination with tolerance analysis, it was assumed that critical sizes of rotors at various stages were in normal distribution within a specified tolerance zone; eccentric errors of assembled rotors at different stages were also predicted by the Monte Carlo method. Yang et al. [14] also probed into how the quantitative distribution of the circumferential assembly orientation affects the cumulative errors of assembly. Jin et al. [15] established a Jacobian-Torsor-matrix-based assembly error analysis model concerned with the partial parallel chains. In essence, all the literature described above fell into a category of tolerance analysis. Depending on the location and orientation tolerance of rotor parts, a multistage rotor assembly error propagation model was constructed by Wang et al. [16] in order to achieve optimal matching for the assembly orientations of the rotors at diverse stages. However, such an approach still had its failure in constructing a uniform objective function. A neural network optimization model was constructed by Sun et al. [17] in order to minimize the eccentricity error and verticality error for a multistage rotor.

In fact, the optimization of the mass attributes of a multistage rotor should be especially highlighted in order to prevent vibration of an aeroengine from exceeding its upper limits. To optimize rotor unbalance, an assembly method was put forward by Liu et al. [18]. However, the center-ofmass coordinate in this study is not obtained by actual measurement but is estimated by assuming a conversion ratio and is based on the centroid coordinates. In order to overcome this defect, Sun et al. [19] made use of a vertical dynamic balancing machine to measure the mass attributes of the rotors at different stages; on this basis, an assembly approach was proposed for the purpose of optimizing both the coaxiality and unbalance.

According to the research status of the assembly optimization of the multistage rotor described above, the research objects were extended from the 2-D [10-14] to the 3 -D rotors [12, 14-19], and from the virtual rotors [10-14] to the actual rotors [15-19]. Regarding their optimization objectives, they were improved from considering rotors' geometric attributes alone [10-17] to taking both geometric and mass attributes into account $[18,19]$. The defects of the existing research methods are summarized from three perspectives of the error propagation model, the assembly optimization strategy, and the optimization algorithm as follows:

(a) The existing assembly error propagation models for multistage rotor did not take into account the alignment process of screw holes of the adjacent rotor (b) It is unreasonable to optimize the mass parameters by using the measurement datum of the geometric parameters as the assembly datum

(c) The existing optimization algorithm is used to calculate the objective function under all possible assembly orientations and to select the optimal assembly orientations by comparing all the results, which is inefficient

Targeted at above problems, a genetic algorithm- (GA-) based assembly optimization method for the multistage of an aeroengine is proposed in this study. In Section 2.1, a spatial location propagation model is developed for the multistage rotor of an aeroengine. The alignment process of the assembly screw holes of the adjacent rotor is considered for the first time. In Section 2.2, a new assembly optimization strategy is proposed to select different assembly datum for the specific values of the coaxiality and unbalance, respectively. In Section 2.3, according to the GA optimization theories, a double-objective fitness function is constructed based on the coaxiality and unbalance. The simulation and experimental results show that the assembly optimization method proposed in this paper can be utilized to achieve synchronous optimization of the coaxiality and unbalance.

\section{Methods}

2.1. Spatial Location Propagation Model. During machining of the single-stage rotors, the coaxiality and parallelism errors are inevitable in the upper and lower mounting surfaces. Under the circumstance that the rigid coupling of rotors at different stages is realized by the precision bolts, the errors of the respective rotors are propagated from one stage to another through the assembly lips. In this way, a cumulative error is generated. Furthermore, the actual spatial location of a rotor at respective stages is deviated from its ideal assembly position. If the actual spatial position can be exactly figured out after assembly of rotors at various stages, the corresponding cumulative error subsequent to the assembly can be also obtained. In Figure 1, an assembly process of the 3-D two-stage rotor is portrayed. Clearly, when centroid of the lower surface is selected as the origin of coordinates during the measurement of rotors at various stages, their assembly process may be described as follows: Bottom mounting surface of Rotor B is rotated by a certain angle, making it parallel to a top mounting surface of Rotor A; afterward, centroid in the bottom mounting surface of Rotor B undergoes translation so that it overlaps with that of the top mounting surface of Rotor A.

Based on the above ideas of assembly, a descending assembly method is raised in this paper. To be specific, assembly processes of $n$ rotors are deemed as those of $n-1$ two-stage rotor; and, each process is fulfilled by a rotation and a translation simultaneously. A descending assembly process of a 3-D four-stage rotor is presented in Figure 2. The assembly process is divided into three steps as follows:

Step-1: the $X$-axis is formed by a projection axis, on the bottom surface, of a line connecting the highest and the lowest points of the top surface of Rotor 3; the bottom 


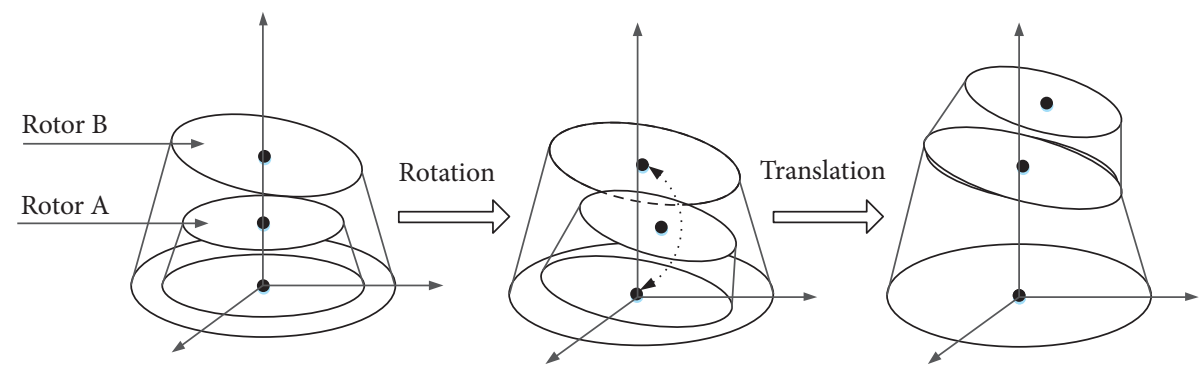

FIgure 1: The assembly process of a two-stage rotor.

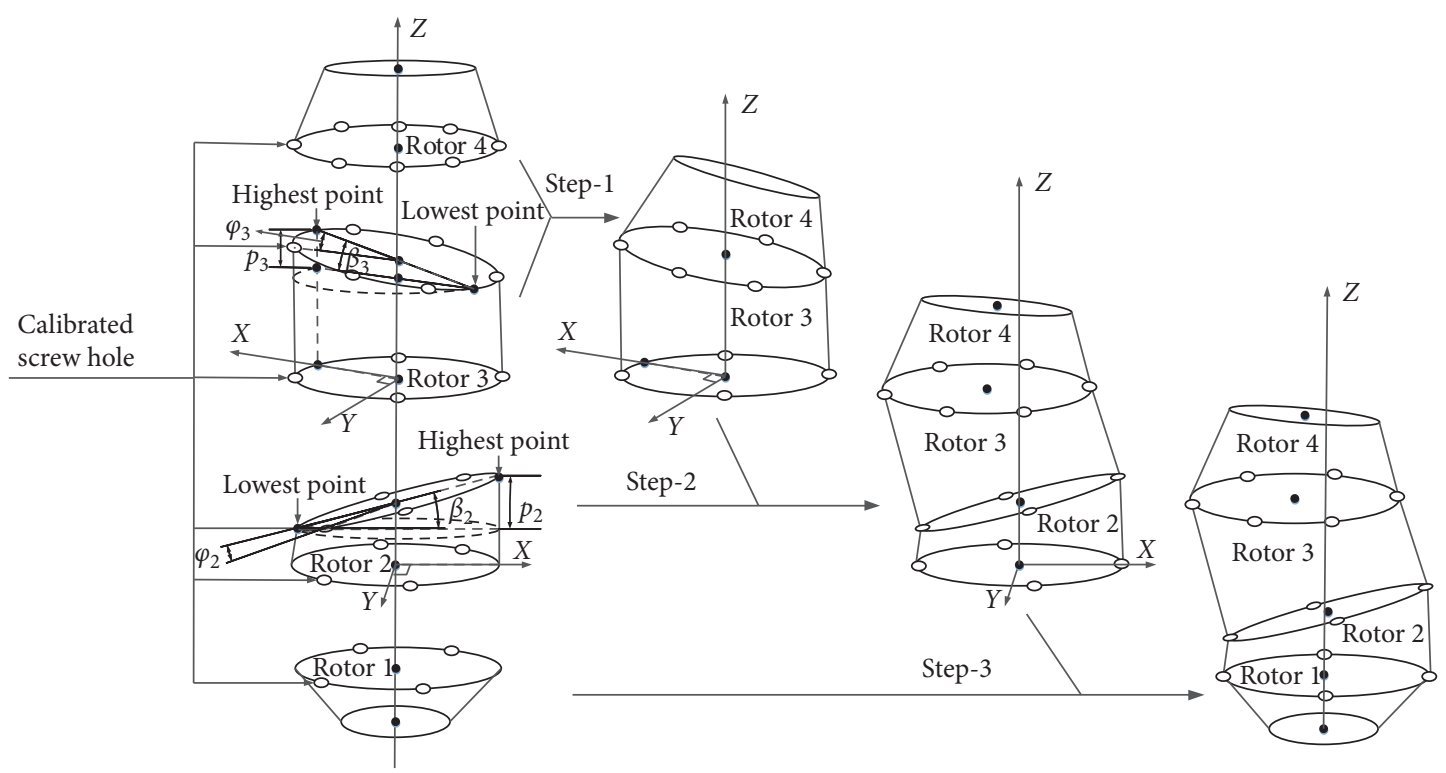

Figure 2: The descending assembly process of a four-stage rotor.

surface is used as a $X Y$-plane and Rotor 4 is rotated by an angle of $\beta_{3}$ around the $Y$ - axis on the above described plane; after that, the center in the bottom surface of Rotor 4 is translated to that in the top surface of Rotor 3. In this way, assembly of Rotors 4 and 3 is completed. Such assembly processes are the same as those shown in Figure 1.

Step-2: an assembly of Rotors 4 and 3 is seen as a single rotor that is further assembled with Rotor 2, so that an assembly process of the two-stage rotor is thus completed. Similarly, a line connecting the highest and the lowest points on the top surface of Rotor 2 is projected on its bottom surface, where its projection axis serves as the $X$-axis. Afterward, the bottom surface of Rotor 2 is selected as $X Y$-plane; and the assembly of Rotors 4 and 3 is rotated by an angle of $\beta_{2}$ around the $Y$-axis on this plane. Then, the center in the bottom surface of Rotor 3 is translated to that in the top surface of Rotor 2 .

Step-3: in this case, an assembly of Rotors 4, 3, and 2 is regarded as a single rotor; together with Rotor 1, a twostage rotor assembly process is fulfilled.

In the following, a case where uniformly distributed screw holes are circumferentially presented in rotors and the feasibility of actual measurement are discussed. The screw holes of an adjacent two-stage rotor must be aligned prior to assembly. After their alignment is achieved, the subsequent mounting and optimization procedures can be fulfilled. In literature [16-19], the geometric parameters of a single-stage rotor are measured by a turntable provided with a 4-channel displacement sensor. For example, the concentricity, parallelism, and other parameters can be obtained once the rotor is rotated by one revolution on the turntable. Although it is simple and efficient, such a measurement method fails to determine the precise location and orientation of the center in the screw hole. Consequently, the angles at which rotors at different stages are rotated during screw holes alignment cannot be identified. Therefore, a 3-D coordinate measurement device should be adopted for the measurement of the geometric parameters related to a single-stage rotor. Firstly, the center in any screw hole needs to be calibrated circumferentially for the rotor, and a phase difference between this center and the highest point should be recorded during the measurement of the parallelism of upper and lower surfaces, such as the angle $\varphi_{3}$ on the top surface of rotor 2 and the angle $\varphi_{2}$ on that of Rotor 2 . Here, it should be noted that the screw holes counts and the uniform distribution angles on the upper mounting surface may differ from those on the lower mounting surface at the time of screw holes design for single-stage rotors. However, there 
should be at least one pair of aligned screw holes existing on the upper and lower mounting surfaces; in other words, they have the same phases. Such a pair of screw holes is selected as the calibrated screw holes, so that the angles by which rotors at diverse stages are rotated during the screw holes alignment can be neglected. The reason is that an angle of the highest points of these rotors relative to their calibrated screw holes remains unchanged. When centroid coordinates, the parallelism of the upper and lower mounting surfaces of rotors at diverse stages, the measured radius, and the highest or lowest point at their top surfaces are measured, all points have the same movement trajectories as far as the rigid rotors are concerned. As expressed in equation (1), the actual coordinates of any point in such a rigid rotor may be worked out for the assembled rotors at various stages.

$$
\mathbf{I}_{n+1}^{\prime}=\mathbf{I}_{n+1}\left[\prod_{n+1:-1: 1}\left(\mathbf{R z}_{n+1} \mathbf{R y}_{n+1}\right)\right]+\mathbf{I}_{n}^{\prime}\left(n \in \mathbf{N}^{*}, n \geq 1\right),
$$

where $\mathbf{I}_{n}=\left[\begin{array}{lll}x_{n} & y_{n} & z_{n}\end{array}\right], \mathbf{I}_{n}^{\prime}=\left[\begin{array}{lll}x_{n}^{\prime} & y_{n}^{\prime} & z_{n}^{\prime}\end{array}\right]$

$$
\begin{aligned}
\mathbf{R z}_{n+1} & =\left[\begin{array}{ccc}
\cos \left[\theta z_{n+1}+\left(\phi_{n+1}-\phi_{n}\right)\right] & -\sin \left[\theta z_{n+1}+\left(\phi_{n+1}-\phi_{n}\right)\right] & 0 \\
\sin \left[\theta z_{n+1}+\left(\phi_{n+1}-\phi_{n}\right)\right] & \cos \left[\theta z_{n+1}+\left(\phi_{n+1}-\phi_{n}\right)\right] & 0 \\
0 & 0 & 1
\end{array}\right] \\
\mathbf{R y}_{n+1} & =\left[\begin{array}{ccc}
\cos \left(\beta_{n+1}\right) & 0 & \sin \left(\beta_{n+1}\right) \\
0 & 1 & 0 \\
-\sin \left(\beta_{n+1}\right) & 0 & \cos \left(\beta_{n+1}\right)
\end{array}\right] \\
\beta_{n+1} & =\tan ^{-1} \frac{p_{n+1}}{d_{n+1}},
\end{aligned}
$$

where $\mathbf{I}_{n}$ refers to a spatial position vector of the $n$th stage rotor before assembly, $\mathbf{I}_{n}^{\prime}$ to that after assembly of the $n$th stage rotor, $\mathbf{R z}_{n+1}$ to a transformational matrix formed by rotation of the $(n+1)$ th stage rotor around axis $Z, \theta z_{n+1}$ to an angle by which the $(n+1)$ th stage rotor rotates around axis $Z$ relative to the $n$th stage rotor, $\mathbf{R y}_{n+1}$ to a transformation matrix produced by rotation of the $(n+1)$ th stage rotor around axis $Y$ at the bottom surface, $\phi_{n}$ to an angle between the center of the calibrated screw hole of the $n$th stage rotor and its highest point, $\beta_{n+1}$ to an angle formed by top and bottom surfaces of the $(n+1)$ th stage rotor, $p_{n+1}$ to parallelism for top and bottom surfaces of the $(n+1)$ th stage rotor as well, and $d_{n+1}$ to a measurement radius of the top surface of the $(n+1)$ th stage rotor. Concerning all the above parameters, only $\theta z_{n+1}$ is a variable, the other parameters can be measured by a 3-D coordinate measuring machine (CMM).

2.2. Assembly Optimization Strategy. Both the coaxiality and unbalance out of tolerance may lead to excessive vibration of an aeroengine. However, their action mechanisms are different from each other. To be concrete, the coaxiality out of tolerance has the chance to cause not only collisions between stators and rotors but also rotor misalignment. As for the unbalance out of tolerance, it can probably produce unbalanced centrifugal force and torque, which exerts an extra dynamic load on the bearing system. Considering this, the assembly optimization of an aeroengine should achieve synchronous optimization of the geometric and mass attributes. In the existing literature, the overall coaxiality and unbalance optimization for the multistage rotor assemblies selects a normal axis across the center in the bottom surface of the bottom rotor as the assembly datum for optimization (that is, the Table axis in Figure 3). Without a doubt, such an assembly strategy has certain shortcomings. In fact, the journals of the front and the back shafts of the rotor are, respectively, positioned on swing frames on left and right sides of a dynamic balancing machine in the course of dynamic balancing subsequent to the rotor assembly. Not only should the corresponding spin axis be approximate to a line connecting midpoints of journals of the front and the back shafts but also the measured radius vector of the eccentric mass needs to be perpendicular to such a spin axis (i.e., the orientation-varying axis in Figure 4). Moreover, the spin axis may vary along with variations in assembly orientations of the rotors at various stages. On this basis, a novel assembly strategy is raised in this paper. By virtue of this strategy, a proper datum can be selected for the assembly optimization in line with the specific values of the coaxiality and unbalance. As shown in Figure 3, while table axis is utilized as the optimization datum for the coaxiality, the orientation-varying axis serves as that for the unbalance mass (i.e., a line of centers in the axle journals of the bottom and the top rotors).

Provided that the table axis acts as an optimization datum for the coaxiality, the coaxiality $C$ of an $n$-stage rotor after assembly can be expressed in equation (3). Therefore, the maximum concentricity of the assembled rotors at various stages can be denoted as follows: 


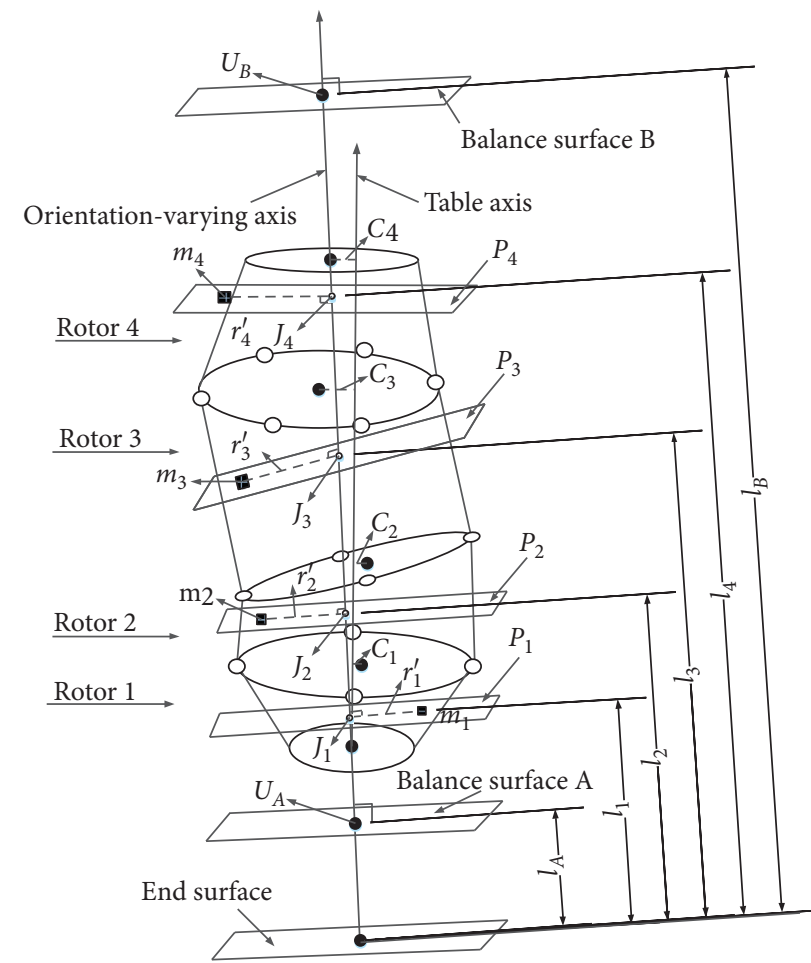

FIgURE 3: The assembly optimization datum of a four-stage rotor.

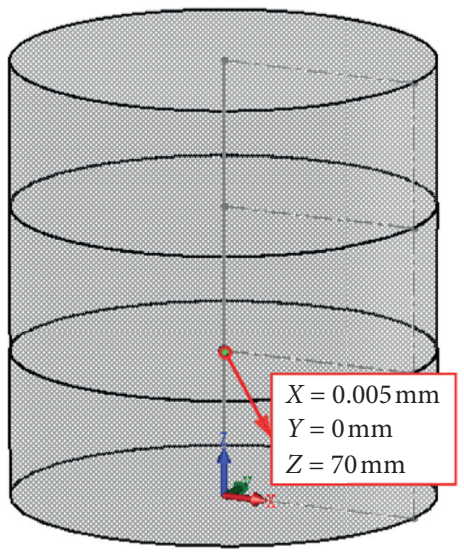

(a)

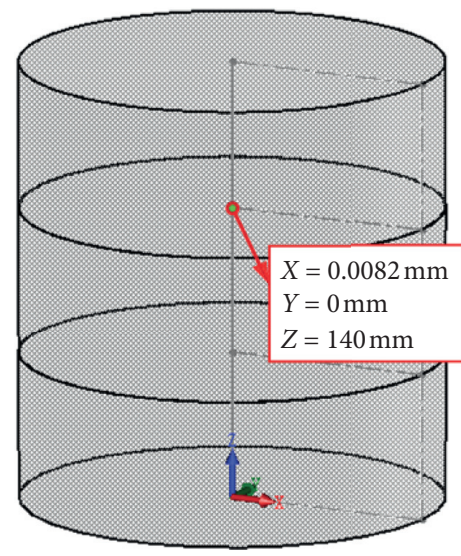

(b)

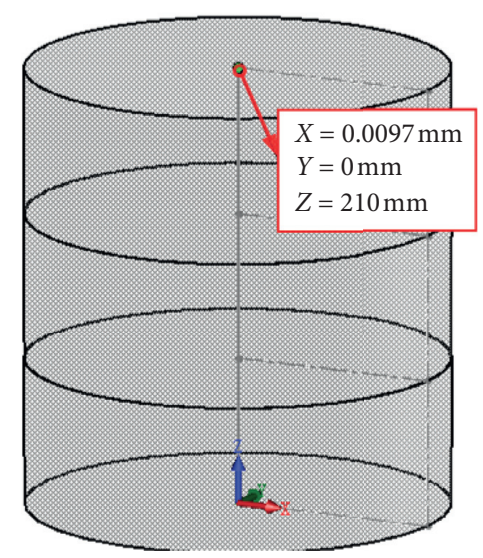

(c)

Figure 4: The centroid coordinates of the top surface of the assembled 3-stage rotor are measured by software. (a) The 1st rotor. (b) The 2nd rotor. (c) The 3rd rotor.

$$
C=\sqrt{\sum_{i=1}^{n}\left(\frac{C_{i}}{\bar{C}_{n}}\right)^{\varepsilon} \varepsilon \bar{C}_{n}}
$$

where $\bar{C}_{n}$ is the mean value of concentricity obtained from $n$ rotors that have been assembled, denoted by $\bar{C}_{n}=\left(\sum_{i=1}^{n} C_{i} / n\right)$, and $C_{n}$ represents the concentricity after assembly of the $n$th stage rotor. Here, the comparison procedures of the concentricity values for all rotors are substituted by equation (3). Once a value of $\varepsilon$ is high enough, the computed result is just the maximum concentricity of the multistage rotor. Moreover, $C_{n}$ is expressed in another equation as follows:

$$
C_{n}=2 \sqrt{e_{n x}^{\prime 2}+e_{n y}^{\prime 2}}
$$

where $\mathbf{E}_{n}^{\prime}=\left[\begin{array}{lll}e_{n x}^{\prime} & e_{n y}^{\prime} & e_{n z}^{\prime}\end{array}\right], \mathbf{E}_{n}=\left[\begin{array}{lll}e_{n x} & e_{n y} & e_{n z}\end{array}\right], \mathbf{E}_{n}^{\prime}$ refers to the coordinates of centroid subsequent to the top assembly surface for the $n$th stage rotor, $\mathbf{E}_{n}$ measured by a 3-D CMM stands for those before top assembly surface for the $n$th stage rotor. Here, the value of $\mathbf{E}_{n}^{\prime}$ can be obtained by substituting $\mathbf{E}_{n}$ into equation (1).

Once the optimization datum for the unbalance is selected to be the orientation-varying axis, the unbalance of the assembled rotors at various stages is firstly obtained through the equation (5), that is, 


$$
\mathbf{U}_{n}=m_{n} \mathbf{r}_{n}^{\prime}
$$

where $\mathbf{U}_{n}$ is the unbalance of the $n$th stage rotor assembled, that is, a product of $m_{n}$ (i.e., the unbalance mass of the $n$th stage rotor) and $\mathbf{r}_{n}^{\prime}$ (i.e., the action radius vector of the $n$th stage rotor). As for $\mathbf{r}_{n}^{\prime}$, it is solved by the following steps:

Step-1: a linear equation expressing the orientationvarying axis can be written as follows:

$$
\frac{x-x_{0}}{e_{n x}^{\prime}-x_{0}}=\frac{y-y_{0}}{e_{n y}^{\prime}-y_{0}}=\frac{z-z_{0}}{e_{n z}^{\prime}-z_{0}}=t_{n}
$$

where $\left(x_{0}, y_{0}, z_{0}\right)$ is a position vector of the bottom center of the bottom rotor. Under the circumstance that the table axis serves as the datum for the measurement of the rotor's geometric parameters, such a point is the origin of the coordinates system by default.

Step-2: as for the plane $P_{n}$ that is extended across an unbalance mass point of the $n$th stage rotor and perpendicular to the orientation-varying axis, it is expressed in the equation as follows:

$$
\begin{aligned}
& \left(e_{n x}^{\prime}-x_{0}\right)\left(x-r_{n x}^{\prime}\right)+\left(e_{n y}^{\prime}-y_{0}\right)\left(y-r_{n y}^{\prime}\right) \\
& \quad+\left(e_{n z}^{\prime}-z_{0}\right)\left(z-r_{n z}^{\prime}\right)=0,
\end{aligned}
$$

where $\mathbf{R}_{n}^{\prime}=\left[\begin{array}{lll}r_{n x}^{\prime} & r_{n y}^{\prime} & r_{n z}^{\prime}\end{array}\right], \mathbf{R}_{n}=\left[\begin{array}{lll}r_{n x} & r_{n y} & r_{n z}\end{array}\right]$, where $\mathbf{R}_{n}^{\prime}$ represents the coordinate of the assembled $n$th stage rotor based on the unbalance mass point; $\mathbf{R}_{n}$ measured by a dynamic balancing machine stands for coordinates of the unbalance mass point obtained before the assembly of the $n$th stage rotor. By substituting $\mathbf{R}_{n}$ into equation (1), $\mathbf{R}_{n}^{\prime}$ is solved.

Step-3: the coordinates of an intersecting point between the orientation-varying axis and the plane $P_{n}$ are expressed in the following Equation.

Firstly, equations (6) and (7) form a simultaneous equation. Through such a simultaneous equation, the parameter $t_{n}$ in the linear equation expressing the orientation-varying axis can be obtained:

$$
t_{n}=\frac{\left(e_{n}^{\prime}-x_{0}\right)\left(r_{n x}^{\prime}-x_{0}\right)+\left(e_{n y}^{\prime}-y_{0}\right)\left(r_{n y}{ }^{\prime}-y_{0}\right)+\left(e_{n z}^{\prime}-z_{0}\right)\left(r_{n z}^{\prime}-z_{0}\right)}{\left(e_{n x}^{\prime}-x_{0}\right)^{2}+\left(e_{n y}^{\prime}-y_{0}\right)^{2}+\left(e_{n z}^{\prime}-z_{0}\right)^{2}}
$$

By substituting $t_{n}$ into equation (6), the coordinates of the intersection point $J_{n}$ are acquired, where $J_{n}=\left[\begin{array}{lll}j_{n x} & j_{n y} & j_{n z}\end{array}\right]$.

Step-4: the action radius of the unbalance mass relative to the orientation-varying axis, that is, the modulus of $\mathbf{r}_{n}^{\prime}$, is expressed in the following equation:

$$
r_{n}^{\prime}=\sqrt{\left(j_{n x}-r_{n x}^{\prime}\right)^{2}+\left(j_{n y}-r_{n y}^{\prime}\right)^{2}+\left(j_{n z}-r_{n z}^{\prime}\right)^{2}} .
$$

By substituting equation (9) into equation (5), a value of $\mathbf{U}_{n}$ is acquired. After that, unbalance masses of the rotors at diverse stages need to be decomposed into balance surfaces $A$ and $B$ through equation (10).

$$
\left\{\mathbf{U}_{A}=\sum_{j=1}^{n} \frac{l_{B}-l_{j}}{l_{B}-l_{A}} \mathbf{U}_{j}, \mathbf{U}_{B}=\sum_{j=1}^{n} \frac{l_{j}-l_{A}}{l_{B}-l_{A}} \mathbf{U}_{j},\right.
$$

where $\mathbf{U}_{A}$ and $\mathbf{U}_{B}$ turn out to be the unbalance of the balancing surfaces $A$ and $B$, respectively. In this context, the maximum unbalance of the $n$-stage rotor can be obtained by the following equation:

$$
\mathbf{U}=\sqrt[\varepsilon]{\left(\frac{\mathbf{U}_{A}}{\bar{U}_{n}}\right)^{\varepsilon}+\left(\frac{\mathbf{U}_{B}}{\bar{U}_{n}}\right)^{\varepsilon}} \bar{U}_{n}
$$

where $\bar{U}_{n}$ is the mean value of $\mathbf{U}_{A}$ and $\mathbf{U}_{B}$. The resolution principle of equation (11) is the same as that of $C$, substituting procedures of unbalance comparison for balancing surfaces $A$ and $B$.
Furthermore, a double-objective optimization function is established based on the coaxiality and unbalance derived out for the assembled $n$-stage rotor:

$$
\left\{\begin{array}{l}
V-\min F(x)=\min [C(x) U(x)]^{T}, \\
x=\left(\theta_{z 1}, \theta_{z 2}, \ldots \ldots, \theta_{z n}\right), \theta_{z n} \in\left(0,180^{\circ}\right),
\end{array}\right.
$$

where the design variable $x$ represents a vector formed by assembly orientations of the $n$-stage rotor. In addition, $\theta_{z n}$ stands for a rotation angle of the $n$th stage rotor around axis $Z$ of its assembly feature surface, and $C(x)$ and $U(x)$ for single-objective optimization functions, respectively, based on the coaxiality and unbalance.

Specific to the multiobjective optimization problem, the optimal values $f_{\Delta}$ of all objective functions and corresponding optimal points $x_{\Delta}$ are worked out. Generally, it is difficult to obtain optimal solutions simultaneously for all objective functions. In other words, no optimal solution $x^{*}$ can be obtained, enabling all objective functions to reach their own optimal values. Therefore, the ideal point $x^{*}$ cannot be gained for the objective function $F(x)$. However, if the objective functions can approach their own ideal values to the greatest extent, a comparatively satisfactory noninferior solution may be obtained. On this basis, a multiobjective optimization problem is transformed into an issue of solving extremums of single-objective evaluation functions. Furthermore, a weight coefficient $W_{i}$ can be introduced, making each single-objective function dimensionless. Here, the double-objective optimization function is created as follows: 


$$
\left\{\begin{array}{l}
V-\min G(x)=W_{1}\left(C(x)-C_{\Delta}\right)^{2}+W_{2}\left(U(x)-U_{\Delta}\right)^{2}, \\
x=\left(\theta_{z 1}, \theta_{z 2}, \ldots \ldots, \theta_{z n}\right), \theta_{z n} \in\left(0,180^{\circ}\right)
\end{array}\right.
$$

where $C_{\Delta}=\min (C(x)), U_{\Delta}=\min (U(x)), W_{1}=1 / C_{\Delta}$, and $W_{2}=1 / U_{\Delta}$. Such an evaluation function makes the doubleobjective dimensionless, which not only considers that each objective is as close to their ideal value as possible but also reflects that respective objectives are equally important in the entire double-objective optimization issue.

2.3. Genetic Optimization Algorithm. As a global optimization algorithm, GA is aimed at mapping solution space to genetic space, encoding each set of possible solutions into a chromosome. Firstly, a series of candidate solutions are randomly generated and they form an initial population; based on a fitness function designed in advance according to the objective function, fitness of each individual to problemsolving environment is calculated. Then, corresponding selections are made in consistency with the obtained fitness, suppressing chromosomes with low fitness, but promoting those with low fitness. After that, genetic manipulations such as crossover and mutation are performed for the purpose of evolving the next-generation population. Through repeated operations described above, constant evolution toward optimized solutions is achieved. At last, except for a population that meets corresponding convergence conditions and is most adaptive to the problem-solving environment, the optimal solution is also obtained. Depending on the above thoughts of genetic optimization, the double-objective optimization function, expressed in equation (13), derived in Section 2.2, is further developed to a GA fitness function:

$$
\left\{\begin{array}{l}
\text { fitness_G } G(x)=\frac{\left(U(x)-U_{\Delta}\right)^{2}}{U_{\Delta}}+\frac{\left(C(x)-C_{\Delta}\right)^{2}}{C_{\Delta}}, \\
\text { s.t. } x=\left(\theta_{z 1}, \theta_{z 2}, \ldots, \theta_{z n}\right), \theta_{z n} \in\left(0,180^{\circ}\right) .
\end{array}\right.
$$

Here, the single-objective GA fitness function based on the coaxiality is expressed as

$$
\left\{\begin{array}{l}
\text { fitness_ } C(x)=C(x), \\
\text { s.t. } x=\left(\theta_{z 1}, \theta_{z 2}, \ldots \ldots, \theta_{z n}\right), \theta_{z n} \in\left(0,180^{\circ}\right) .
\end{array}\right.
$$

As for the single-objective GA fitness function based on the unbalance, it is expressed in the following formula:

$$
\left\{\begin{array}{l}
\text { fitness_ } U(x)=U(x), \\
\text { s.t. } x=\left(\theta_{z 1}, \theta_{z 2}, \ldots, \theta_{z n}\right), \theta_{z n} \in\left(0,180^{\circ}\right) .
\end{array}\right.
$$

In terms of GA, its steps are detailed as follows:

Step-1 (initial population): the $n$ elements in an assembly orientation sequence $x$ are regarded as $n$ genes, and each gene varies from $0^{\circ}$ to $180^{\circ}$. Each chromosome is composed of $n$ genes. In this way, 1,000 chromosomes are randomly generated and act as an initial population.
Step-2 (individual evaluation): fitness of each chromosome is figured out for individual evaluation.

Step-3 (selection): chromosomes with the optimal fitness are selected and take the place of a chromosome of the worst fitness.

Step-4 (mutation): purpose of mutation is to improve the current chromosome. According to equation (17), the chromosome with higher fitness has a smaller variation range. As for chromosomes with lower fitness, their variation ranges are larger accordingly.

$$
x_{\text {new }}=x\left[1 \pm \gamma\left(1-\frac{f}{f_{\text {best }}}\right)^{2}\right]
$$

where $x_{\text {new }}$ and $x$ are chromosomes after/before mutation, respectively. $\gamma$ is a random number between 0 and 1 , and $f$ stands for the fitness of $x$ while $f_{\text {best }}$ stands for the optimal fitness of the present population.

Step-5 (crossover): some genes are randomly selected from two chromosomes for replacement and recombination. In this way, a new chromosome is generated. By means of crossover, search capability of GA can be enhanced.

Step-6 (termination criteria): the algorithm is ended when fitness of optimal individuals and the population ceases to rise, and the number of iterations reaches the design value.

\section{Simulation}

3.1. Validation of the Spatial Location Propagation Model. To verify the computational accuracy of the spatial location propagation model given in Section 2.1, the geometric parameters of the 3-stage rotor presented in Literature [16] are used for reference to simulation. On this basis, the angles between the calibration screw hole and the highest point is $0^{\circ}$ by default (i.e. $\phi_{1}=\phi_{2}=\phi_{3}=0$ ). In addition, SolidWorks, the 3-D software of drawing, was utilized to reconstruct the corresponding 3 -stage rotor model. Through a measurement function of the software, the centroid coordinates of the assembled rotors at various stages were measured and then compared with the computation results achieved by the model.

Provided that the initial orientations of the rotors at various stages turn out to be $0^{\circ}$, that is, in the case of $\theta_{z 1}=\theta_{z 2}=\theta_{z 3}=0^{\circ}$, the centroid coordinates of the top surface of the assembled 3-stage rotor are presented in Table 1 . They are highly consistent with measurement results produced by software, as shown in Figure 4. This indicates that, in the context where the geometric parameters of these rotors have been known, the proposed spatial location propagation model has capability of accurately predicting the spatial locations of the assembled rotors at various stages.

3.2. Genetic Optimization. It is assumed that the 3-stage simulation rotor described in Section 3.1 is made of steel. Furthermore, the mass attributes (see Table 2) of the rotors 
TABLE 1: The centroid coordinates of the top surface of the assembled 3-stage rotor.

\begin{tabular}{lcr}
\hline Stage & Simulation results $(\mathrm{mm})$ & Measurement results $(\mathrm{mm})$ \\
\hline 1 & $(0.005,0,70)$ & $(0.005,0,70)$ \\
2 & $(0.083,0,140)$ & $(0.082,0,140)$ \\
3 & $(0.098,0,210)$ & $(0.097,0,210)$ \\
\hline
\end{tabular}

TABLE 2: The mass parameters of the 3-stage rotor are measured by software.

\begin{tabular}{lcr}
\hline Stage & Mass $(\mathrm{kg})$ & Centroid coordinates $(\mathrm{mm})$ \\
\hline 1 & 17.2630 & $(0.0036,0,35)$ \\
2 & 17.2630 & $(0.0036,0,35)$ \\
3 & 17.2630 & $(0.0036,0,35)$ \\
\hline
\end{tabular}

at different stages were measured by software. Then, the optimization simulation of the assembly orientations was conducted by GA based on the three strategies of the coaxiality-based single-objective optimization (Strategy-1), the unbalance-based single-objective optimization (Strategy-2), and the double-objective optimization based on the coaxiality and unbalance (Strategy-3). In terms of the 1 st stage rotor, its assembly orientation was designed at $0^{\circ}$ by default; each gene was designed to hold two genes, that is $\left(\theta_{z 1}, \theta_{z 2}\right)$, and the number of initial populations was set at 1,000 . In addition, the selection principle of the internal parameters of GA referred to the literature [20], then the mutation rate, crossover rate, and iterative times were set as $0.01,0.9$, and 500 , respectively. Under the circumstance that a case where connecting screw holes are present in the rotors at various stages is ignored, the corresponding simulation results are as those given in Table 3. The convergence progress of the single-objective optimization for the coaxiality is shown in Figure 5, and the optimal fitness reaches $0.0058 \mathrm{~mm}$. The convergence progress of the singleobjective optimization for the unbalance is shown in Figure 6 , and the optimal fitness reaches $32.2568 \mathrm{~g} \cdot \mathrm{mm}$. The convergence progress of the double-objective optimization for the coaxiality and unbalance is shown in Figure 7, and the dimensionless optimal fitness reaches 0.0012. Meanwhile, the coaxiality and unbalance are $0.0064 \mathrm{~mm}$ and $32.2574 \mathrm{~g} \mathrm{~mm}$, respectively. From Figures 5-7, it can be seen that all the optimization processes for the above three optimization objects have a good convergence within 500 iterations.

The unbalance obtained by the double-objective genetic optimization is almost the same as that of the single-objective optimization based on the unbalance, and the optimization effect of the coaxiality is increased by $25 \%$. Clearly, the genetic optimization based on the double-objective has the potential to preferably realize synchronous optimization of the coaxiality and unbalance, and their optimization outcomes are closer to those of the single-objective optimization.

In the practical assembly procedures, the rigid connection of the rotors at various stages is achieved through precision bolts. The screw holes are discretely distributed, enabling available assembly orientations to be discrete as well. In this case, the initial populations are also discrete variables. Therefore, the optimal alignment relations of the screw holes in the rotors at various stages should be figured out through the optimal solution based on the secondary calculation. Firstly, the angles of the screw holes adjacent to the optimal solution are determined to calculate all possible alignment relations (number: $2^{n-1}$ ) of the rotors at different stages. From these relations, the optimal alignment of screw holes can be selected. Relative to calibrated screw holes of the $(n-1)$ th stage rotor, the number of intervals among screw holes into which the $n$th stage rotor is rotated can be directly identified. As assumed, there are 24 circumferential screw holes on the upper and lower assembly surfaces of each rotor, and the distribution angle of screw holes is set at $15^{\circ}$.

The sampling angle between the center of the calibrated screw hole and the highest point of each rotor is designed to be $0^{\circ}$. Under these circumstances, the optimization simulation in a case of no screw holes is repeated. Regarding relevant simulation results, they are listed in Table 4 . Moreover, an optimal solution must be selected in the context where the angle of screw holes is formed.

3.3. Monte Carlo Simulation. In the process of the actual measurement, certain measurement errors, such as a system error or an error caused by the corresponding measuring means, may be inevitably incurred. The present paper further probes into the influence of the measurement errors on the genetic optimization results. It is assumed that the geometric parameters of the 3-stage simulation rotor all conform to a normal distribution with a standard deviation of $\sigma / 6$, where values of all parameters are those presented in Section 3.1, and $\sigma$ stands for the tolerance values of these parameters. For specific settings of such tolerance values, they are presented in Table 5. Based on the Monte Carlo method, the double-objective genetic optimization is simulated 10,000 times.

During 10,000 iterations by virtue of the Monte Carlo method, the genetic optimization results are distributed in a range of $159.62^{\circ} \sim 180^{\circ}$ as far as the assembly orientations of a rotor at the 2 nd stage is concerned, as shown in Table 6 . Moreover, their mean values and standard deviations turn out to be $179.88^{\circ}$ and $0.2798^{\circ}$, respectively (see Figure 8 ). Probability for the optimal values of $\theta_{z 2}$ to lie between $179^{\circ}$ and $180^{\circ}$ is calculated to be $99.527 \%$, signifying a highly 
TABLE 3: The simulation results of the genetic optimization without screw holes.

\begin{tabular}{|c|c|c|c|c|}
\hline $\begin{array}{l}\text { Assembly } \\
\text { strategy }\end{array}$ & $\begin{array}{l}\text { The optimal solution of the 2nd stage } \\
\qquad\left({ }^{\circ}\right)\end{array}$ & The optimal solution of the 3 rd stage $\left(^{\circ}\right)$ & $\begin{array}{l}\text { Coaxiality } \\
(\mathrm{mm})\end{array}$ & $\begin{array}{l}\text { Unbalance } \\
\text { (g.mm) }\end{array}$ \\
\hline Strategy-1 & 147.5016 & 157.9014 & 0.0058 & 60.8752 \\
\hline Strategy-2 & 180 & 180 & 0.0066 & 32.2568 \\
\hline Strategy-3 & 179.9671 & 179.9877 & 0.0064 & 32.2574 \\
\hline
\end{tabular}

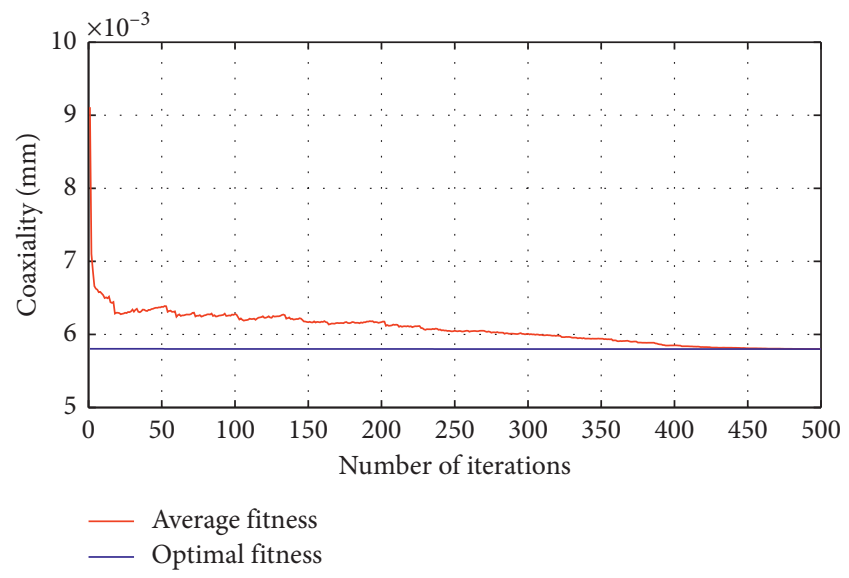

FIGURE 5: The convergence progress of the single-objective optimization for the coaxiality.

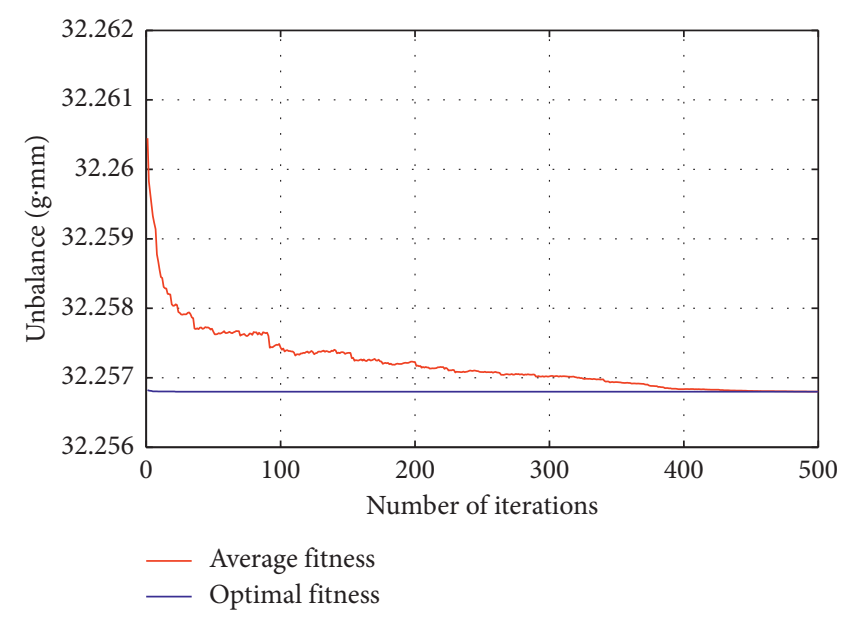

FIGURE 6: The convergence progress of the single-objective optimization for the unbalance.

concentrated distribution. As for the assembly orientations of a rotor at the 3 rd stage, the distribution range, the mean value, and the standard deviation of their genetic optimization results are proved to be, respectively, $145.91^{\circ}, 179.89^{\circ}$ and $0.7174^{\circ}$ (see Figure 9). Similarly, the probability for optimal values of $\theta_{z 3}$ to lie between $179^{\circ}$ and $180^{\circ}$ is $98.594 \%$, indicating a highly concentrated distribution as well. Clearly, no large prediction errors are incurred in genetic optimization results after parameter measurement errors of rotors are taken into account. In addition, the results of genetic optimization based on the double-objective optimization functions remain significantly consistent and accurate.

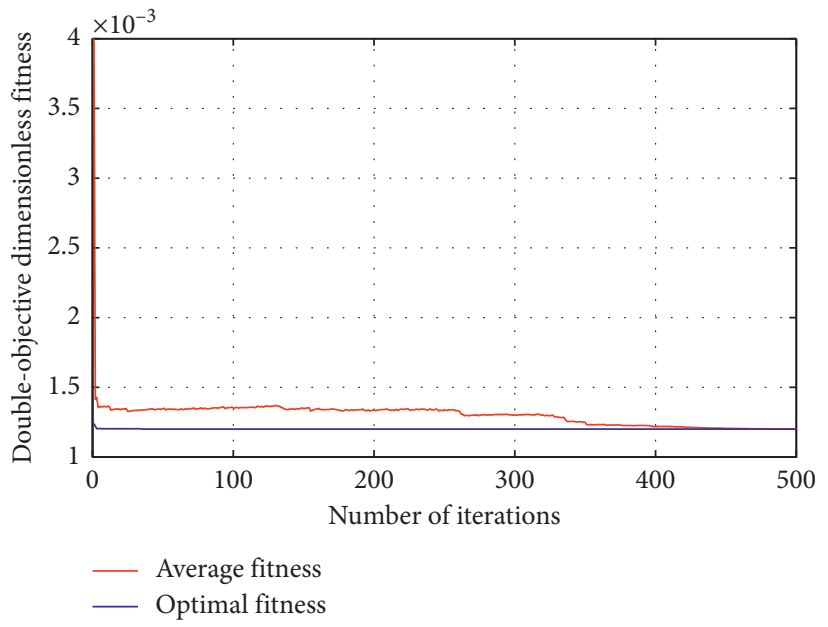

Figure 7: The convergence progress of the double-objective optimization for the coaxiality and unbalance.

\section{Experiments}

Following, an aeroengine was scaled down to the original proportion and simplified into a 4-stage simulation rotor. A section view of such a rotor system has been shown in Figure 10. As observed, it is composed of a front shaft, a LPC, an HPC, and a back shaft. To further prove the validity of the proposed assembly optimization method here, experiments are performed and the corresponding experimental steps are detailed below:

Step-1: the geometric parameters of rotors at diverse stages were measured by a 3-D CMM.

As presented in Figure 11(a), the geometric parameters of the front shaft are separately measured. First, the central position of any screw hole in the circumferential direction was calibrated. A connecting line between the center of this screw hole and a symmetric axis of the front shaft served as the $X$-axis. After that, not only the coordinates of the centroid in a supporting section holding diameters of the front shaft were measured, but the centroid was calibrated as the origin of the coordinate system. Subsequently, the following parameters of the top mounting surface were measured, that is, the coordinates of the centroid, the parallelism errors, the angle of the highest point relative to the center of the calibrated screw hole, and the height. Regarding measurement principles of the LPC, the HPC, and the back shaft, they are the same as those adopted by the front shaft. Moreover, the selected 3-D CMM is manufactured by Carl Zeiss AG, and its measurement 
TABLE 4: The simulation results of the genetic optimization with screw holes.

\begin{tabular}{lcccc}
\hline $\begin{array}{l}\text { Assembly } \\
\text { strategy }\end{array}$ & $\begin{array}{c}\text { The optimal solution of the 2nd stage } \\
\left({ }^{\circ}\right)\end{array}$ & The optimal solution of the 3rd stage $\left(^{\circ}\right)$ & $\begin{array}{c}\text { Coaxiality } \\
(\mathrm{mm})\end{array}$ & $\begin{array}{c}\text { Unbalance } \\
(\mathrm{g} \cdot \mathrm{mm})\end{array}$ \\
\hline Strategy-1 & 150 & 165 & 0.0058 & 54.4719 \\
Strategy-2 & 180 & 180 & 0.0066 & 32.2568 \\
Strategy-3 & 165 & 165 & 0.0064 & 42.9476 \\
\hline
\end{tabular}

TABLE 5: The tolerance values of the geometric parameters of the rotors at diverse stages.

\begin{tabular}{lccccc}
\hline Stage & Eccentricity $(\mu \mathrm{m})$ & Parallelism $(\mu \mathrm{m})$ & Measured radius $(\mu \mathrm{m})$ & Height $(\mu \mathrm{m})$ & Sampling angle of the highest point $\left(^{\circ}\right)$ \\
\hline 1 & 4 & 4 & 4 & 4 & 2 \\
2 & 4 & 4 & 4 & 4 & 2 \\
3 & 4 & 4 & 4 & 4 & 2 \\
\hline
\end{tabular}

Table 6: The Monte Carlo simulation results.

\begin{tabular}{lcccc}
\hline Optimal solution & Maximum value $\left({ }^{\circ}\right)$ & Minimum value $\left(^{\circ}\right)$ & Mean value $\left(^{\circ}\right)$ & Standard deviation $\left(^{\circ}\right)$ \\
\hline$\theta z_{2}$ & 180 & 159.62 & 179.88 & 0.2798 \\
$\theta z_{3}$ & 180 & 145.91 & 179.89 & 0.7174 \\
\hline
\end{tabular}

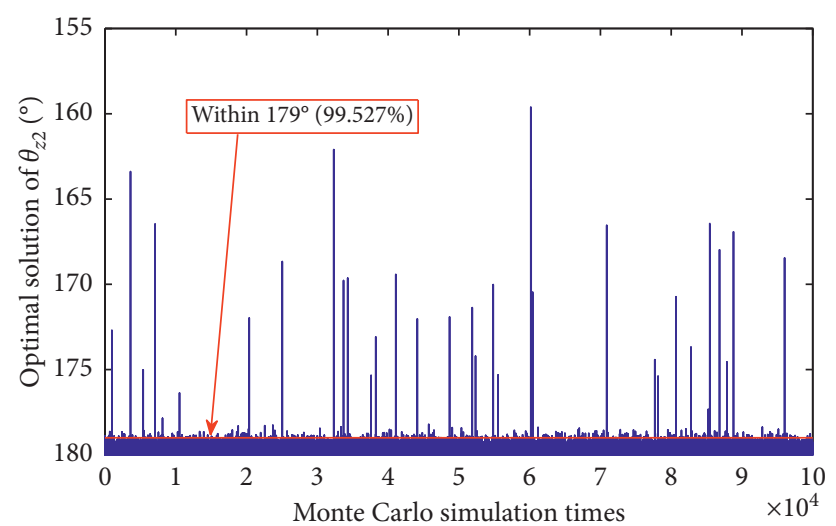

FIGURE 8: The distribution of the genetic optimization results for a rotor at the 2nd stage.

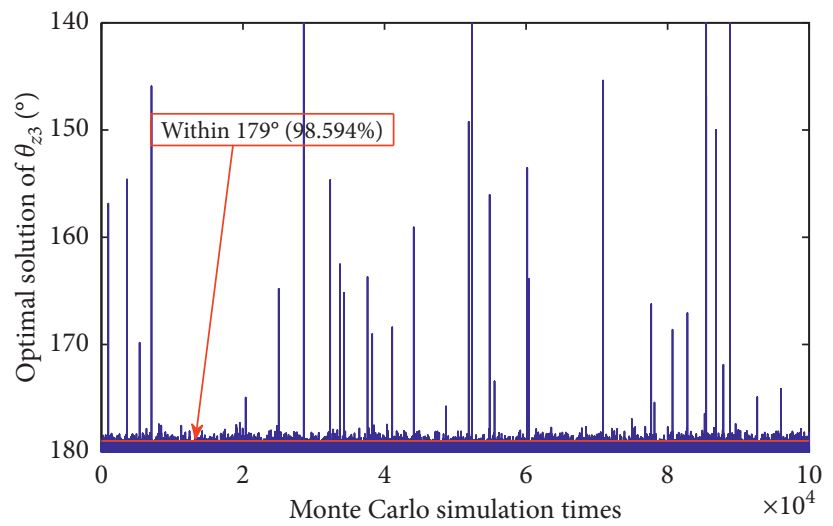

Figure 9: The distribution of the genetic optimization results for a rotor at the 3rd stage.

accuracy reaches $2 \mu \mathrm{m}$. The geometric parameters of the 4-stage rotor are presented in Table 7.

Step-2: the mass parameters of rotors at diverse stages were measured by a dynamic balancing machine.
As shown in Figure 11(b), the unbalance of the front shaft was independently measured. In addition, the horizontal datum and the horizontal measuring surface were selected as the two planes of the output unbalance, 


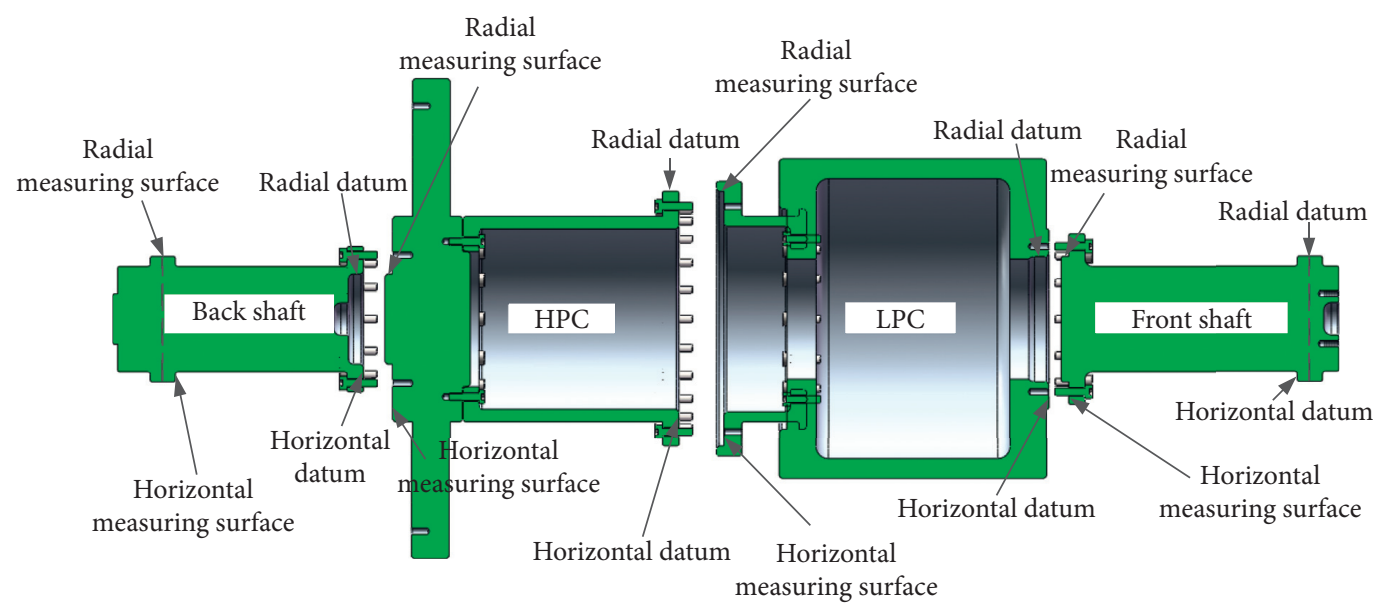

(a)

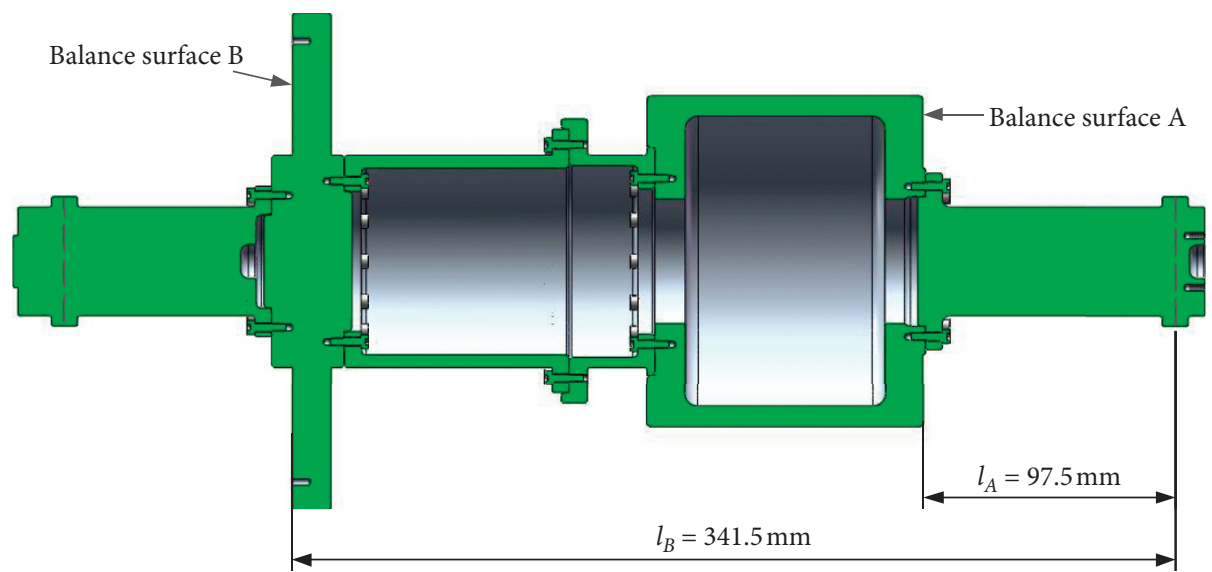

(b)

FIgURE 10: The section view of the 4-stage rotor. (a) Exploded view. (b) Assembled view.

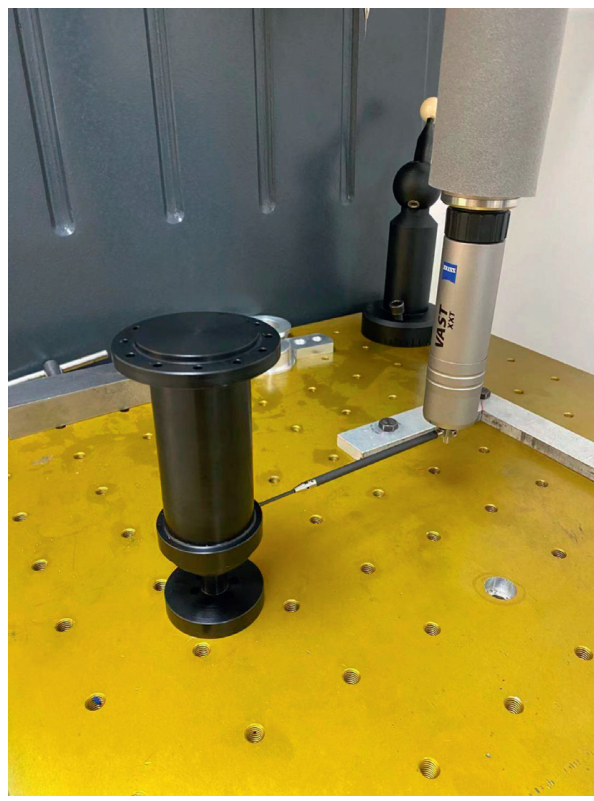

(a)

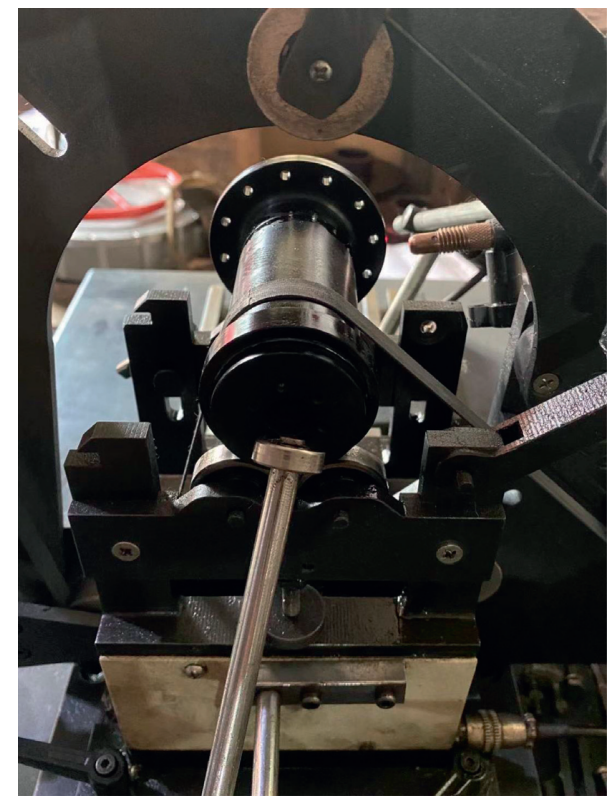

(b)

FIgURE 11: The setup picture for measuring the geometric and mass parameters of the front shaft. (a) The geometric parameters are measured by a CMM. (b) The mass parameters are measured by a dynamic balancing machine. 
TABLE 7: The geometric parameters of the 4-stage rotor.

\begin{tabular}{|c|c|c|c|c|c|c|}
\hline Component & $\begin{array}{l}\text { Centroid coordinates } \\
\text { of top assembly } \\
\text { surface }\left(\mathbf{I}_{n}\right)(\mathrm{mm})\end{array}$ & $\begin{array}{l}\text { Measuring } \\
\text { radius }\left(d_{n}\right) \\
\quad(\mathrm{mm})\end{array}$ & $\begin{array}{l}\text { Parallelism } \\
\left(p_{n}\right)(\mathrm{mm})\end{array}$ & $\begin{array}{l}\text { Angle between the } \\
\text { calibrated screw hole } \\
\text { and the highest point } \\
\left(\phi_{n}\right)\left({ }^{\circ}\right)\end{array}$ & $\begin{array}{c}\text { Number of } \\
\text { distributed screw } \\
\text { holes (top surface) }\end{array}$ & $\begin{array}{c}\text { Number of } \\
\text { distributed screw } \\
\text { holes (bottom } \\
\text { surface) }\end{array}$ \\
\hline Front shaft & $(0.0021,0.0108,96.5)$ & 25 & 0.015 & 230 & 12 & 12 \\
\hline LPC & $(0.0156,0.0036,138)$ & 51 & 0.019 & 68 & 24 & 12 \\
\hline HPC & $(-0.0179,0.0019,115)$ & 18 & 0.020 & 49 & 12 & 24 \\
\hline Back shaft & $(-0.0121,-0.0047,80)$ & 25 & 0.011 & 152 & 12 & 12 \\
\hline
\end{tabular}

TABLE 8: The mass parameters of the 4-stage rotors.

\begin{tabular}{lcccc}
\hline Component & $\begin{array}{c}\text { Radial datum } \\
\text { Unbalanced mass } \\
\left(m_{n}\right)(\mathrm{g})\end{array}$ & $\begin{array}{c}\text { Coordinates of unbalance mass point } \\
\left(\mathbf{R}_{n}\right)(\mathrm{mm})\end{array}$ & $\begin{array}{c}\text { Radial measuring surface } \\
\text { Conbalanced mass } \\
\left(m_{n}\right)(\mathrm{g})\end{array}$ & $\begin{array}{c}\text { Coordinates of unbalance mass point } \\
\left(\mathbf{R}_{n}\right)(\mathrm{mm})\end{array}$ \\
\hline Front shaft & 0.17 & $(24.9657,-1.3084,0)$ & 0.1 & $(23.1796,9.3652,96.5)$ \\
LPC & 1.97 & $(8.1392,23.6380,0)$ & 1.41 & $(33.4590,38.4902,138)$ \\
HPC & 1.10 & $(-50.7206,-5.3310,0)$ & 2.34 & $(-8.7266,15.7432,115)$ \\
Back shaft & 0.08 & $(2.5051,-17.8248,0)$ & 0.16 & $(17.9835,17.3665,80)$ \\
\hline
\end{tabular}

and the center of the calibrated screw hole during the measurement of the geometric parameters was taken as the initial phase point. The measured double-sided unbalance vectors were transformed into the form of unbalanced mass acting on the outer circle and coordinates of unbalance mass point. With regard to the LPC, the HPC, and the back shaft, their measurement principles are identical to those of the front shaft. Moreover, the dynamic balancing machine (the minimum residual unbalance: $0.5 \mathrm{~g} \cdot \mathrm{mm} / \mathrm{kg}$ ) selected for use is manufactured by Shanghai Dongyijing Testing Machineries Co., Ltd. The mass parameters of the 4stage rotor are presented in Table 8.

Step-3: according to the measured values of the geometric and mass parameters, the four-stage rotor was optimized for three times, including:

(a) Coaxiality based on the single-objective optimization (Strategy-1)

(b) Unbalance based on the single-objective optimization (Strategy-2)

(c) Double-objective optimization based on the coaxiality and unbalance (Strategy-3)

Step-4: according to the three assembly optimization strategies of Step-3, the corresponding optimal assembly orientations were calculated, and the rotors were assembled in consistency with the three optimization results, and both the coaxiality and unbalance of the assemblies were measured (see Figure 12).

\section{Results and Discussion}

It is demonstrated by the relevant experimental results (see Table 9 and Figure 13) that the coaxiality achieved by the double-objective optimization increases by $12.7 \%$ if compared with that generated by the coaxiality-based singleobjective optimization; however, the unbalance drops by
$33 \%$ accordingly. As for the double-objective optimization, the unbalance and coaxiality thus achieved, respectively, rise and reduce by $32 \%$ and $38.6 \%$ in comparison with those produced by the unbalance-based single-objective optimization. These phenomena show that the proposed doubleobjective genetic optimization algorithm is capable of realizing synchronous optimization of the coaxiality and unbalance of a multistage rotor. In addition, they also prove the validity of making the fitness function dimensionless.

Furthermore, the proposed rotor assembly optimization method is concerned with three aspects. First, a spatial location propagation model needs to be constructed. According to relevant simulation results, such a model is able to accurately predict the actual spatial positions of the assembled rotors at various stages. In contrast to the existing studies, a factor of the screw holes for assembly is directly introduced into the model for the first time, which is more suitable for the actual measurement related to the multistage rotor of an aeroengine and also delivers guidance on rotor assembly. The second aspect is concerned with the assembly optimization strategies. Considering the actual operating conditions of rotor unbalance, two-plane unbalance generated by the dynamic balancing machine must be perpendicular to the spin axis of the corresponding measured rotors. Moreover, such a spin axis is just a line connecting midpoints of the front and back shafts of the rotor. Regarding the unbalance optimization investigated in the existing studies, the corresponding assembly datum is the same as that selected for the coaxiality optimization. In other words, a normal axis across the bottom center of the bottom rotor serves as the datum. Moreover, the normal axis is also the datum based on which geometric parameters of the single-stage rotors are measured. However, when such an axis is used as the datum for the unbalance optimization, it is inconsistent with the measurement conditions of the dynamic balancing machine on the one hand; on the other hand, the corresponding experimental results have no 


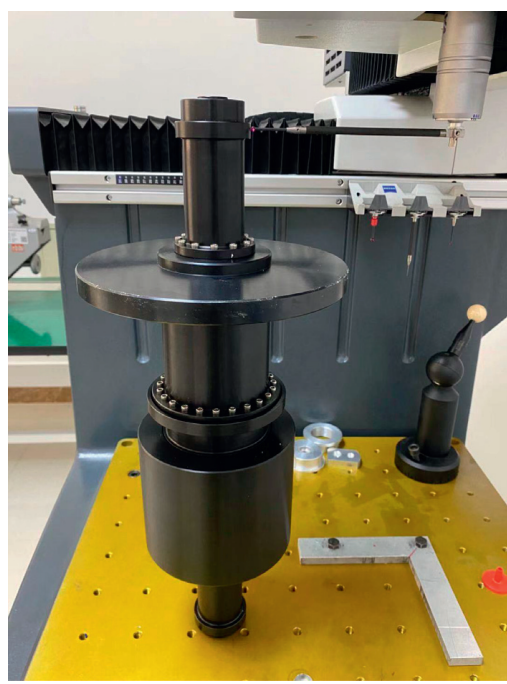

(a)

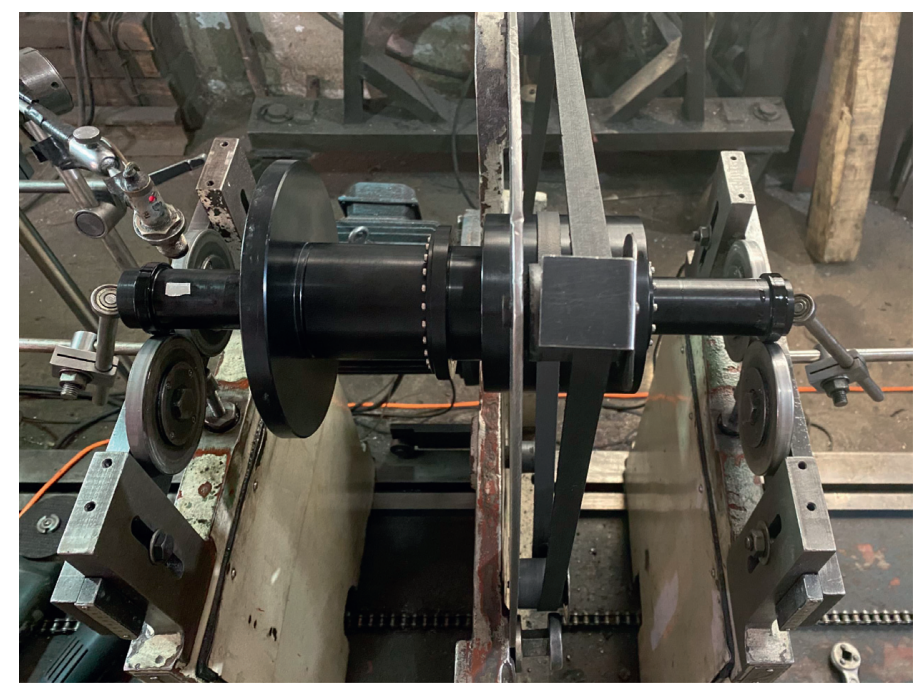

(b)

FIGURE 12: The setup picture for measuring the geometric and mass parameters of the four-stage rotor. (a) The geometric parameters are measured by CMM. (b) The mass parameters are measured by dynamic balancing machine.

Table 9: The experimental results of the 4-stage rotor.

\begin{tabular}{lccccc}
\hline $\begin{array}{l}\text { Assembly } \\
\text { strategy }\end{array}$ & $\begin{array}{c}\text { The optimal solution of the } \\
\text { 2nd stage }\left(^{\circ}\right)\end{array}$ & $\begin{array}{c}\text { The optimal solution of the } \\
\text { 3rd stage }\left(^{\circ}\right)\end{array}$ & $\begin{array}{c}\text { The optimal solution of the } \\
\text { 4th stage }\left({ }^{\circ}\right)\end{array}$ & $\begin{array}{c}\text { Coaxiality } \\
(\mathrm{mm})\end{array}$ & $\begin{array}{c}\text { Unbalance } \\
(\mathrm{g} \cdot \mathrm{mm})\end{array}$ \\
\hline Strategy-1 & 30 & 15 & 180 & 0.074 & 133 \\
Strategy-2 & 30 & 60 & 150 & 0.136 & 67 \\
Strategy-3 & 30 & 30 & 120 & 0.086 & 89 \\
\hline
\end{tabular}

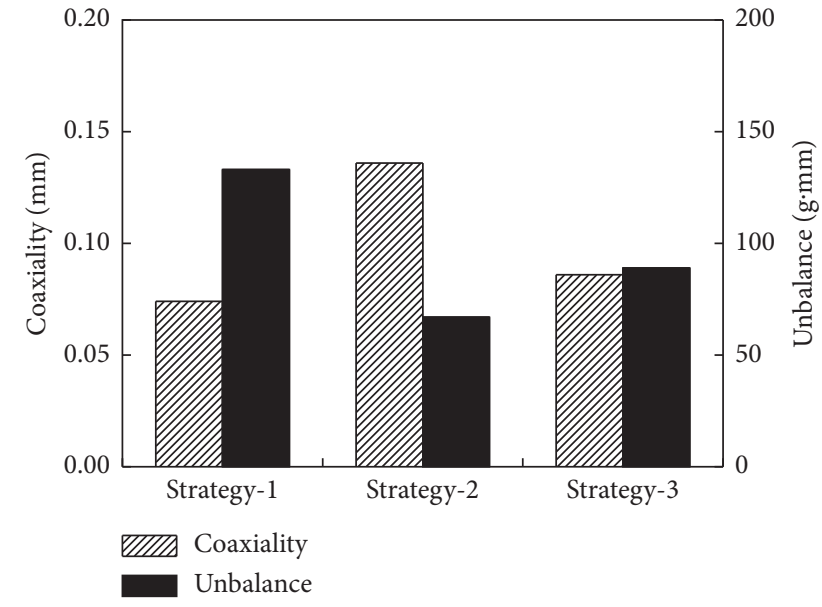

Figure 13: The histogram of the experimental results.

reference significance. Therefore, the proposed assembly strategy here is to select different types of the assembly datum for the various values of the coaxiality and unbalance, ensuring consistency with the actual measurement conditions. In terms of the optimization algorithm, the bionics algorithm is now seldom applied in assembly optimization. Here, GA is employed to achieve optimal matching of the assembly orientations for the rotors at different stages. Such a strategy is applicable not only for the optimization of the continuous angles but also for the optimization of discrete angles in the presence of screw holes for assembly.

\section{Conclusions}

Due to limitations of the current level of industry development, neither machining error nor material defect can be avoided during manufacturing of an aeroengine. The errors of the individual rotors at diverse stages are constantly propagated and accumulated during assembly. It is urgent to put forward an assembly instruction method that can be used to accurately predict the optimal alignment relation among rotors at different stages on the premise of the known geometric and mass parameters of these rotors. In this study, a GA-based assembly optimization method is developed for the multistage rotor of an aeroengine. Main tasks related to it can be described through the following three aspects:

(1) A spatial location propagation model was developed for the multistage rotor. The alignment process of the assembly screw holes of the adjacent rotors was considered for the first time. On the basis of a descending assembly method, the assembly procedures of the $n$-stage rotor were simplified into those of $n$-1 two-stage rotor. Provided that the geometric and mass parameters of each single-stage rotor are given, the coaxiality and unbalance of the assemblies 
can be accurately predicted in any alignment relation of the screw holes.

(2) A new assembly optimization strategy was proposed to select different assembly data for the specific values of the coaxiality and unbalance, respectively. More particularly, a normal axis across the bottom center of the bottom rotor acted as the assembly datum for the coaxiality optimization and line of centers in supporting sections of the journals of the front and the back shaft was used as that for the unbalance optimization. Such an assembly optimization strategy is more suitable for a multistage rotor as far as their actual measurements and operating conditions are concerned.

(3) The synchronous optimization of the coaxiality and unbalance was achieved by using GA. In addition, the optimal assembly orientations of the rotors at different stages were also identified thus. It was further demonstrated by the Monte Carlo simulation that, even if certain measurement errors are incurred, the genetic optimization results still show high consistency and reliability.

\section{Data Availability}

The modeling data used to support the findings of this study are available from the corresponding author upon request.

\section{Conflicts of Interest}

The authors declare that they have no conflicts of interest.

\section{Acknowledgments}

This work was supported by the Outstanding Youth Project of Natural Science Foundation of Heilongjiang Province, under Grant No. JQ2019E002. The authors are grateful for the financial support.

\section{References}

[1] W. Sun, T. Li, D. Yang et al., "Dynamic investigation of aeroengine high pressure rotor system considering assembly characteristics of bolted joints," Engineering Failure Analysis, vol. 112, Article ID 104510, 2020.

[2] S. Ding, X. Zheng, J. Bao et al., "A comprehensive study of three dimensional deviation analysis methods for aero-engine rotors assembly," IOP Conference Series: Materials Science and Engineering, vol. 688, Article ID 033039, 2019.

[3] Y. Sun, J. Guo, J. Hong, G. Liu, W. Wu, and C. Yue, "Repair decision based on sensitivity analysis for aero-engine assembly," International Journal of Precision Engineering and Manufacturing, vol. 20, no. 3, pp. 347-362, 2019.

[4] M. Wang, Q. Han, B. Wen, H. Zhang, and T. Guan, "Modal characteristics and unbalance responses of fan rotor system with flexible support structures in aero-engine," in Proceedings of the Institution of Mechanical Engineers, Part G: Journal of Aerospace Engineering, vol. 231, no. 9, pp. 1686-1705, 2017.

[5] M. Thiele and G. Heins, "Computationally efficient method for identifying manufacturing induced rotor and stator misalignment in permanent magnet brushless machines,"
IEEE Transactions on Industry Applications, vol. 52, no. 4, pp. 6109-6116, 2015.

[6] A. K. Verma, S. Sarangi, and M. H. Kolekar, "Misalignment fault detection in induction motor using rotor shaft vibration and stator current signature analysis," International Journal of Mechatronics and Manufacturing Systems, vol. 6, no. 5-6, pp. 422-436, 2013.

[7] P. M. Hai and P. Bonello, "A computational parametric analysis of the vibration of a three-spool aero-engine under multifrequency unbalance excitation," Journal of Engineering for Gas Turbines \& Power, vol. 133, Article ID 072504, 2011.

[8] X. Hu, F. Gao, C. Cui et al., "Analysis of mass unbalance torque on a spinning superconducting rotor," IEEE Transactions on Applied Superconductivity, vol. 24, no. 3, pp. 1-4, Article ID 3600204, 2014.

[9] S. W. Wang, R. Mo, and H. C. Yang, "Quantitative and meticulous methods to aero-engine assembly process," Applied Mechanics and Materials, vol. 220-223, no. 3, pp. 206209, 2012.

[10] T. Hussain, Z. Yang, A. A. Popov et al., "Straight-build assembly optimization: a method to minimize stage-by-stage eccentricity error in the assembly of axisymmetric rigid components (two-dimensional case study)," Journal of Manufacturing Science and Engineering, vol. 133, no. 3, Article ID 031014, 2011.

[11] Z. Yang, A. A. Popov, S. McWilliam et al., "Variation propagation control in mechanical assembly of cylindrical components," Journal of Manufacturing Systems, vol. 31, no. 2, pp. 162-176, 2012.

[12] Z. Yang, T. Hussain, A. A. Popov, and S. McWilliam, "Novel optimization technique for variation propagation control in an aero-engine assembly," in Proceedings of the Institution of Mechanical Engineers, Part B: Journal of Engineering Manufacture, vol. 225, no. 1, pp. 100-111, 2010.

[13] Z. Yang, T. Hussian, A. A. Popov et al., "A comparison of different optimization techniques for variation propagation control in mechanical assembly," IOP Conference, vol. 26, Article ID 012017, 2011.

[14] T. Hussain, G. Yasinshaikh, and S. A. Shaikh, "Variation propagation control in straight-build assemblies: $2 \mathrm{D}$ case study," Mehran University Research Journal of Engineering \& Technology, vol. 32, no. 1, pp. 71-80, 2013.

[15] S. Jin, S. Ding, Z. Li et al., "Point-based solution using Jacobian-Torsor theory into partial parallel chains for revolving components assembly," Journal of Manufacturing Systems, vol. 46, pp. 46-58, 2017.

[16] L. Wang, C. Sun, J. Tan, B. Zhao, and G. Wan, "Improvement of location and orientation tolerances propagation control in cylindrical components assembly using stack-build assembly technique," Assembly Automation, vol. 35, no. 4, pp. 358-366, 2015.

[17] C. Sun, C. Li, Y. Liu et al., "Prediction method of concentricity and perpendicularity of aero engine multistage rotors based on PSO-BP neural network," IEEE Access, vol. 99, p. 1, 2019.

[18] Y. Liu, M. Zhang, C. Sun et al., "A method to minimize stageby-stage initial unbalance in the aero engine assembly of multistage rotors," Aerospace Science and Technology, vol. 85, pp. 270-276, 2018.

[19] C. Sun, Z. Liu, Y. Liu, X. Wang, and J. Tan, “An adjustment method of geometry and mass centers for precision rotors assembly," IEEE Access, vol. 7, pp. 169992-170002, 2019.

[20] X. S. Yang, Nature-Inspired Meta-Heuristic Algorithms, Luniver Press, Beckington, UK, 2008. 